Invited paper

\title{
Recent advances in research and control of malaria, leishmaniasis, trypanosomiasis and schistosomiasis
}

\author{
S. L. Croft, ${ }^{1}$ L. Vivas ${ }^{1}$ and S. Brooker ${ }^{1}$
}

\begin{abstract}
SUMMARY In the Eastern Mediterranean Region of the World Health Organization (WHO), malaria, schistosomiasis, leishmaniasis and trypanosomiasis are the parasitic diseases of major importance. Our review focuses on recent advances in the control and treatment of these diseases with particular reference to diagnosis, chemotherapy, vaccines, vector and environmental control. The Roll Back Malaria Programme, for example, emphasizes the use of insecticide treated bednets in Africa and targets a 30 -fold increase in treated bednet use by 2007. Increasing risk factors for leishmaniasis include urbanization, extended agricultural projects and civil unrest and the increase in patients with Leishmania infantum and HIV co-infection in the Region may signal a new threat. In the past 20 years, human African trypanosomiasis has resurged in sub-Saharan Africa; within the Region it has become more common in the southern Sudan where anthroponotic and zoonotic sub-species infections overlap. Schistosomiasis in the Region is caused by either Schistosoma haematobium or S. mansoni and large-scale control efforts include providing regular treatment to at-risk groups and supporting drug delivery through schools.
\end{abstract}

\section{Introduction}

The continuing burden of parasitic diseases upon the health of the human population worldwide, especially in tropical and developing countries, is clearly demonstrated in the recent estimates of disability adjusted life years (DALY), which incorporate both life lost from premature death and years of life lived with disability due to disease [1]. However, these figures fail to indicate the broader effect of these diseases on the social and economic life of individuals and families. In the Eastern Mediterranean Region of the World Health Organization (WHO), four parasitic diseases-malaria, schistosomiasis, leishmaniasis and trypanosomiasis - are of major importance. The
Small Grants Scheme of the Eastern Mediterranean Regional Office supports research on these parasitic diseases and has given many grants for projects that aim to improve our understanding of the control of these diseases. Our review focuses on recent advances in the control and treatment of these parasitic diseases with particular reference to diagnosis, chemotherapy, vaccines, vectors and environmental control.

\section{Malaria}

Malaria remains one of the most serious public health problems in the world. The annual global burden of malaria is estimated

${ }^{1}$ Department of Infectious and Tropical Diseases, London School of Hygiene and Tropical Medicine, London, United Kingdom.

المجلة الصحية لشرق المتوسط، منظمة الصحة العالمية، المجلد التاسع، العدد ع، ب... 
at 1.1 million deaths, mostly due to Plasmodium falciparum infections in children in sub-Saharan Africa, 300-500 million cases and 44 million DALY [2]. The development of resistance to current antimalarials, insecticide resistant mosquitoes, poor health infrastructure coupled with political instability in malaria endemic countries, world climatic changes and population increase are the main reasons for the increasing trend of malaria cases and mortality $[3,4]$. The burden of malaria and its impact on economic growth is well documented and hampers the effectiveness of malaria control programmes contributing to a vicious circle of poverty and disease [5]. Global programmes to reduce the burden of malaria, therefore, must generate coherent policies that are closely linked to a fight against poverty.

\section{Diagnosis}

Cost-effective interventions require affordable methods for the rapid and accurate diagnosis of malaria to ensure prompt and effective treatment. Although microscopy still remains the gold standard due to its cost, specificity and sensitivity (50 parasites/mL blood) when done by a highly skilled technician, several methods have been developed for the detection of proteins produced by the malaria parasite during development and multiplication [6,7]. Dipstick methods based on immunochromatography for the detection of three parasite proteins, $P$. falciparum histidine-rich protein 2 (HRP2), $P$. falciparum lactate dehydrogenase (pLDH) and plasmodium aldolase, use monoclonal antibodies that specifically recognize these proteins [810].

The addition of a non-species specific pLDH antibody allows the differentiation of $P$. falciparum from non- $P$. falciparum infections $[10,11]$. P. falciparum HRP2 can be detected with methods such ParaSight F (Becton Dickinson, Franklin Lakes, New Jersey, United States of America), ICT Pf/ Pv (BINAX, Portland, Maine, USA), Paracheck-Pf (Orchid Biomedical System, Goa, India) and OptiMAL method for the detection of pLDH (DiaMed, Cressier sur Morat, Switzerland) [10,12]. Of these, OptiMAL and ICT Pf/Pv offer the possibility of detection of non- $P$. falciparum infections by the simultaneous use of a $P$. falciparum specific pLDH antibody and a panspecific pLDH antibody (OptiMAL) or the combination of a P. falciparum-specific HPR-2 antibody with the panspecific antialdose antibody (ICT Pf/Pv). A new prototype of the commercially available ParaSight F + V method, FV99-2 (Becton Dickinson), showed greater sensitivity than the original in studies in Asia and South America and is also able to detect $P$. vivax infections [13]. The NOW ${ }^{\circledR}$ ICT (BINAX) method, a new version of the ICT Pf/Pv, showed improved performance over previous versions in Thailand [14]. These tests are limited by differences in sensitivity and specificity between non-endemic and endemic areas, high costs compared with microscopy and false positive results due to the persistence of parasite proteins in the circulation after parasite clearance, occasional failure to detect high parasite densities and interbatch variability $[6,10]$.

Polymerase chain reaction (PCR) methods are very sensitive $(<5$ parasites $/ \mu \mathrm{L}$ blood) and useful for species differentiation, but cannot be used in remote areas, are expensive and can give false positive results due to the persistence of DNA in the circulation despite parasite clearance [10]. Gene amplification methods using micrototal analysis systems are in development for use in environments that lack infrastructure and could be cheaply mass-produced [7]. Whether these new technologies will im-

المجلة الصحية لشرق المتوسط، منظمة الصحة العالمية، المجلد التاسع، العدد ع، ب...T 
prove upon existing methods remains to be seen.

\section{Control of transmission}

The Roll Back Malaria Programme emphasizes the introduction of insecticide treated bednets in Africa for vector control and the target is a 30-fold increase in treated bednet use in the 5 years from 2002. Despite the initial suggestion that measures that reduce parasite transmission in highly endemic areas may lead to a change in both the clinical spectrum of severe disease and the overall burden of severe malaria morbidity by reducing the level of immunity, the benefits of the introduction of insecticide treated bednets have been clearly demonstrated by a reduction in morbidity and mortality that is independent of the level of malaria transmission $[15,16]$. Use and willingness to purchase insecticide treated bednets are determined by socioeconomic status [17]. To increase coverage and reduce inequality in access to treated nets it will be necessary to improve affordability or provide this service free of charge as part of an overall poverty reduction strategy $[18,19]$. Several programmes are under way to expand insecticide treated bednet use involving the provision of sustained subsidies, tariff reduction and synergy between public and private sectors [20]. Until these financial issues are resolved, treated bednet use will fail to fully benefit the most needy. Residual insecticide spraying has been as effective as treated bednets in reducing malaria morbidity and mortality but the nets remain the most cost-effective option [21].

\section{Vaccines}

Progress in malaria vaccine development has been slow over the past 30 years partly because the complexity of the parasite was underestimated [22]. Current vaccine can- didates include recombinant antigens derived from pre-erythrocytic, erythrocytic or asexual stages of the parasite that have limited efficacy in clinical trials. The only vaccine strategy that has provided a high level of protection against homologous and heterologous challenge (>90\%) of 10.5 months duration was the immunization of human volunteers with the bites of irradiated mosquitoes [23].

A recent initiative to produce sporozoites in large scale for vaccination has met with scepticism [24,25]. The initiative is appropriate given the limited protection (40\%-70\%) that most recombinant vaccine candidates have afforded in different trials but the logistical barriers are probably insurmountable. The most promising preerythrocytic recombinant vaccine candidate based on the fusion of hepatitis $B$ surface antigen DNA to DNA encoding the circumsporozoite protein RTS,S only afforded 30\%-60\% protection in human volunteers and $47 \%$ in semi-immune adults in the Gambia [26,27]. Other strategies involve a heterologous prime boost immunization in which a circumsporozoite protein DNA vaccine (VCL-2510) is followed by RTS,S [28]. Pre-erythrocytic vaccine candidates following this approach and under initial safety clinical evaluation include multiple epitope-thrombospondin related adhesion protein (ME-TRAP) [27].

Erythrocytic stages are being targeted by vaccination with recombinant merozoite surface proteins (MSP-1, MSP-2 and MSP-3) and glutamate rich protein (GLURP) as invasion blocking vaccines. However, the protection achieved in several clinical studies in different malaria transmission areas using MSP-1 or the MSP-1 containing synthetic SPf66 peptide has been rather low. Blocking sequestration by immunization with $P$. falciparum erythrocyte membrane protein-1 (PfEMP1) is at

المجلة الصحية لشرق المتوسط، منظمة الصحة العالمية، المجلد التاسع، العدد ؟، ب... 
an early experimental stage. Transmission blocking vaccines involving the recombinant gametocyte antigens, Pfs25, are not yet under clinical evaluation [27]. It is clear that the development of new and safe adjuvants could potentially improve the efficacy of vaccination.

\section{Drugs}

The Roll Back Malaria strategy for prompt and effective treatment is a critical factor to reduce mortality due to untreated $P$. falciparum infections in Africa. Particular attention has been given to artemisinin-based combination therapies (ACT) to improve clinical efficacy and to delay the development of resistance. Implementation of this policy is hampered by the varying levels of drug resistance in each country, by the high costs of artemisinin and its derivatives and by the lack of adequate funding for ACT from the Global Fund to countries in which other treatment options are already ineffective $[20,29]$. Of particular importance is the provision of effective treatment for pregnant women and, although no evidence of adverse effects has been documented, further studies on the safety and efficacy of the use of ACT as part of intermittent preventive therapy in the first trimester of pregnancy in areas where there is sulfadoxine-pyrimethamine resistance are a priority [30,31]. Intermittent preventive treatment of children during the first year of age with sulfadoxine-pyrimethamine or amodiaquine administered at the same time as routine childhood vaccinations has been shown to reduce the incidence of severe anaemia and of clinical episodes of malaria significantly [32].

The safety and efficacy of different ACT for the treatment of malaria are being evaluated mainly in Africa and include artemether with lumefantrine, artesunate with mefloquine, artesunate with sulfadoxine and pyrimethamine, artesunate with amodiaquine, dihydroartemisinin with mefloquine and artesunate with atovaquone and proguanil [33] and more recently Lapdap [chlorproguanil/dapsone plus artesunate (CDA)] [34]. Because ACT are considered the most effective treatments for uncomplicated malaria in areas of multidrug resistance, countries that have switched to ACT as part of their malaria control programmes should receive adequate funds. These funds will need to increase substantially if the Roll Back Malaria target to halve malaria deaths by 2005 is to be achieved.

The limited efficacy of currently available antimalarials against drug resistant parasites has led to the recognition of the need to invest in drug discovery. The main current initiative to aid the discovery and development of new and improved antimalarial drugs is the Medicines for Malaria Venture (MMV), a not-for-profit public and private partnership that aims to produce a new drug every 5 years. Current drugs at different stages of development funded by MMV include: OZ 277, a new synthetic peroxide; artemisone, a semisynthetic endoperoxide; isoquine, an improved aminoquinoline; intravenous artesunate for severe malaria; pyronaridine-artesunate combination; paediatric artemether with lumefantrine; DB289, a dicationic molecule based on the activity of pentamidine to treat African sleeping sickness; and dihydroartemisinin-piperaquine combination [34].

The desired profile of new antimalarials is an oral curative treatment in 3 days of multidrug-resistant malaria at low cost that is safe for use in children and pregnant women [35]. Currently twelve MMV funded discovery projects are in exploratory, lead identification and/or optimization stages against different $P$. falciparum targets that must meet the desired profile. Current discovery projects include: glyceraldehyde-

المجلة الصحية لشرق المتوسط، منظمة الصحة العالمية، المجلد التاسع، العدد ؟، ب.. 
3-phosphate dehydrogenase inhibitors (GAPDH); fatty acid biosynthesis inhibitors (FAB 1 and FAS II); peptide deformylase inhibitor (PDF); cysteine protease inhibition (falcipains); dihydrofolate reductase inhibitor; protein farnesyltransferase inhibitors (Pf-PFT); manzamine alkaloids; pyridones; new dicationic molecules; novel tetracycline derivatives; and 8-aminoquinolines [34]. Although the process from drug discovery to deployment can take at least 10 years, the identification of new drug targets through the malaria genome and the commitment from academia and the pharmaceutical industry will undoubtedly speed up this process [35].

\section{Leishmaniasis}

Leishmaniasis is a disease complex with cutaneous and visceral manifestations caused by over 17 species of the protozoan Leishmania parasite. An estimated 12 million humans are infected and the incidence is 0.5 million cases of the visceral form of the disease and 1.5-2.0 million cases of the cutaneous form of the disease. These figures are probably underestimated because leishmaniasis is a reportable disease in only 40 of 88 endemic countries worldwide [36].

In the Region, visceral leishmaniasis (VL) is caused by both $L$. infantum and $L$. donovani and cutaneous leishmaniasis (CL) is caused by L. major, L. tropica and L. aethiopica. Increasing risk factors for leishmaniasis include urbanization, extended agricultural projects and civil unrest [37]. The epidemics of anthro- ponotic VL from $L$. donovani in the Southern Sudan and CL in Afghanistan from L. tropica have been associated with civil unrest. The increase of patients with L. infantum and HIV co-infection in the Region has brought about a new threat. The 2 infections exac- erbate each other and the disease becomes more difficult to treat and can be transmitted by needles shared by drug users $[38,39]$.

\section{Diagnosis}

Good diagnosis underpins understanding of disease prevalence, impact and control. Despite attempts to introduce PCR and other molecular techniques into the diagnosis of CL, direct microscopy of tissue biopsies or biopsy culture techniques remain the most accessible and widely used for this disease [40]. For VL, invasive spleen or bone marrow biopsy methods might be replaced by serological methods that detect antibodies, for example DAT (using freeze dried antigen), the K39 plasma dipstick (using recombinant antigen, now commercially available), or for antigens using the urine dipstick Katex test [41]. The sensitivity and specificity of some of these methods have been recently compared; the antigen test offered some advantages as antibody responses could be variable, especially in HIV co-infections, and present among the asymptomatic or treated and cured [42].

\section{Drugs}

Despite recent improvements, there remains a limited number and type of drugs available for the treatment of leishmaniasis. The standard pentavalent antimonials, sodium stibogluconate and meglumine antimoniate, for both the visceral and cutaneous forms of the disease require long courses by parenteral administration and have variable efficacy. Two important issues regarding antimonials have been drug resistance and cost. A generic formulation of sodium stibogluconate manufactured in India, for example, has been as effective as the product from western companies at 1/14th of the price. In Bihar state, India, north of the Ganges, up to $60 \%$ of VL cases failed to

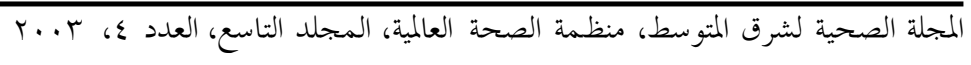


respond to antimonial treatment [43]. Tests on isolates of $L$. donovani from patients who did and did not respond to antimonials, suggest that this is acquired resistance to antimonials [44].

The second-line drugs, amphotericin B and the less frequently used pentamidine, have the additional problem of toxicity. Amphotericin B has been a standby during this developing crisis of antimony resistance [45]. A liposomal formulation of amphotericin B, AmBisome ${ }^{\circledR}$ (Gilead, Great Abington, United Kingdom) has proved to be an excellent treatment for VL and significantly less toxic than the parent drug, but is far too expensive for use in developing endemic countries, even in a single dose treatment [46]. Improved treatment has come through the development of the first orally available drug for VL, miltefosine. In a series of trials, this drug at doses of 100 mg for 28 days achieved a $94 \%$ cure rate, including cure of antimony resistant cases [47]. Miltefosine was registered for treatment of VL in India in March 2002. Another oral drug for VL, the 8-aminoquinoline sitamaquine, is also in clinical development. Finally, the aminoglycoside paromomycin has proved effective in phase II trials and is now in phase III trials in India [48]. VL and HIV co-infection is difficult to treat with a greater than $60 \%$ failure rate following treatment with most anti-leishmanial drugs used either alone or in combination [38]. Highly active antiretroviral therapy (HAART) has some effect on the relapse rate [49].

For CL, a number of uncontrolled clinical trials have suggested that antifungal azoles, for example, itraconazole and fluconazole, have some efficacy. Other drugs, e.g. oral miltefosine, have also been tried [50]. There remains an interest in the development of topical formulations offering improved compliance and reduced cost for treatment of simple CL. The paromomycin (12\% with methyl benzethonium chloride) formulation was most successful, although it was more irritating than a paromomycin formulation containing urea that has been in trial in the Islamic Republic of Iran and Tunisia [51]. Another approach has been the use of drug combinations of a standard pentavalent antimonial with an immunomodulator, most recently with a topical formulation of imiquimod, an antiviral formulation marketed as Aldara (3M Health Care Ltd., Loughborough, United Kingdom) [52].

\section{Vaccines}

The success of leishmanization using a live vaccine, which has been a successful measure in control of CL in the Region, raises ethical issues. However, this practice continues in the absence of defined protein vaccines. Many believe that parasite persistence is required to produce immunity and the Islamic Republic of Iran is producing batches of $L$. major vaccine candidates under internationally accepted Good Manufacturing Practises (GMP). Considerable progress has been made in the production of protein vaccines, both in the production of recombinant peptides and the identification of suitable adjuvants such as CpG oligonucleotides. However, neither VL nor CL candidates have yet reached the clinical trial stage [53].

\section{Control of transmission}

The female sandfly vector (Phlebotomus spp.) has traditionally been controlled by insecticide spraying, recently with pyrethroids used in household spraying. Some sandflies feed indoors where bednets significantly reduce transmission, e.g. in Nepal, where a 70\% reduction in likelihood to develop VL was reported. More recently, pyrethroid-impregnated bednets were used

المجلة الصحية لشرق المتوسط، منظمة الصحة العالمية، المجلد التاسع، العدد ع، ب... 
in the Islamic Republic of Iran, the Syrian Arab Republic, Afghanistan and the Sudan; all showed reductions in cases of CL. Another approach has been to control the reservoir, both in the destruction of rodent habitats for CL and dogs for VL. Insecticide dipping or application to dogs has been used but pyrethroid impregnated dogcollars have some of the best long-lasting effects, reducing the odds of infection in both dogs and children in a trial in the Islamic Republic of Iran [54].

\section{Human African trypanosomiasis}

In the past 20 years there has been a resurgence in cases of human African trypanosomiasis (HAT, or sleeping sickness) in sub-Saharan Africa, with an estimated 300 000-500 000 people infected [55,56]. The majority of cases have been in Angola and the Democratic Republic of the Congo, and within the Eastern Mediterranean Region, in southern parts of Sudan. Two Trypanosoma brucei sub-species cause HAT, the anthroponotic $T$. brucei gambiense that causes the more frequent chronic disease in West and Central Africa and the zoonotic T. brucei rhodesiense that causes the more acute disease in East Africa. Although the course of the diseases caused by the two parasites is different, both forms of HAT are characterized by an initial haemolymphatic stage, followed by a second central nervous system stage when the parasites have crossed the blood-brain barrier. In the southern Sudan there appears to be an overlap of the sub-species [57].

\section{Diagnosis}

Early diagnosis is an important part of the control of HAT. Although parasites can initially be detected in the blood and lymph, parasite numbers decrease as the disease progresses and more sensitive diagnostic methods are required. In addition, subspecies cannot be distinguished morphologically. In addition to the traditional methods of direct microscopy, there are several serological tests including the well-tried card agglutination test for trypanosomiasis (CATT). The card agglutination test can detect asymptomatic cases, but there are problems of false-positives due to other infections and variable levels of signal that make interpretation difficult [58]. A recent paper shows that the technique of proteomic signature analysis is highly specific (100\%) and sensitive (98\%) when applied to blood samples of HAT cases from Angola [59]. Although the technique relies upon expensive equipment and will not be useful in rural Africa, novel protein indicators of disease have been identified that could lead to new simpler diagnostic techniques.

\section{Vaccines}

The antigenic variation of bloodstream form T. brucei trypomastigotes has been a barrier to the development of vaccines. Although research has greatly advanced the understanding of the molecular basis of antigen switching and the 1000 genes responsible for encoding variable surface glycoproteins, a vaccine is unlikely in the near future [60].

\section{Drugs}

Treatment of HAT has remained unchanged for over a decade. In 2001 an agreement was reached between WHO and Médecins sans Frontières (MSF) and the pharmaceutical companies Aventis and Bayer to maintain the manufacture and supply for 5 years of pentamidine, suramin, melarsoprol, nifurtimox (for further clinical trials) and eflornithine [61]. Recent studies with melarsoprol have focused on shorter treatment regimes. Eflornithine was registered 
for use in 1990 for second stage T. brucei gambiense disease (but is ineffective against $T$. brucei rhodesiense) and its potential use in oral dosing regimes and in combinations has been studied $[62,63]$.

The only new drug on or near to clinical trial is the diamidine derivative DB289, a pro-drug that can be orally administered. It is currently in Phase II trials for first stage disease in the Democratic Republic of the Congo [61]. Future studies are likely to focus on drug combinations. There are concerns about the high rate of treatment failures to melarsoprol (up to 30\% was reported in northern Uganda) but the basis for the resistance is unclear. However, the spectre of the T. brucei rhodesiense reservoir in cattle that is resistant to veterinary trypanocides with cross-resistance to human drugs has raised concern in some quarters about another source of resistant parasites [57].

\section{Vector control}

Insecticide use has been open to criticism because of its environmental impact, but with focussed spraying of tsetse fly resting sites this approach is still successful and will continue to play a part in disease control although eradication is unlikely [64].

\section{Schistosomiasis}

Infecting some 200 million people worldwide, human schistosomiasis is a chronic disease caused by infection with one of 5 species of parasitic trematodes of the family Schistosomatidae-Schistosoma haematobium, S. intercalatum, S. japonicum, S. mansoni, and S. mekongi. In the Region, 2 species are found: $S$. haematobium and $S$. mansoni. The former, which causes urinary schistosomiasis, is transmitted by $\mathrm{Bu}$ linus spp. snails, which inhabit less permanent water bodies, because they pre- fer in their lifecycle a period of aestivation in mud, during a dry season. The latter is transmitted by Biomphalaria spp. snails, which are aquatic snails that thrive in irrigation canals and along lakeshores, and causes intestinal schistosomiasis. Many individuals infected with schistosomes also harbour multiple helminth infections, including soil-transmitted helminths like Ascaris lumbricoides, Trichuris trichiura and hookworm.

The first obvious clinical sign of S. haematobium infection is blood in the urine; for intestinal schistosomiasis, it is blood in stool. A consequence of the deposition of schistosome eggs in mucosae and tissues, the clinical and life-threatening complications of schistosomiasis include bladder cancer or serious kidney malfunction in $S$. haematobium infection and severe complications of the liver and spleen in intestinal schistosomiasis. In addition to these clinical complications, it is becoming increasingly appreciated that the effects of infection are worse than previously thought and that schistosomiasis, as well as soiltransmitted helminths, exerts subtle, yet significant, insults on the growth, education and productivity of individuals [65]. For example, intense schistosome infection in children may result in poorer short-term memory and slower reaction times and heavy burdens of schistosome infection can be associated with reduced growth $[66,67]$.

\section{Prospect of control}

Control programmes have shown that much clinical morbidity associated with schistosomiasis can be reversed and prevented through repeated and regular chemotherapy [68-71]. Several controlled trials have shown that treatment may also have a positive effect on growth and cognition $[72,73]$. In addition, many challenges

المجلة الصحية لشرق المتوسط، منظمة الصحة العالمية، المجلد التاسع، العدد ؟، ب..r 
relating to large-scale control have been overcome. For example, teachers and other non-health professionals can distribute anthelmintic drugs effectively to school-age children with minimal training [74]. Mass drug administration has been simplified by the use of a 'dose pole' that determines the correct dose from a child's height and a questionnaire of self-reported blood in urine that accurately, rapidly and inexpensively identifies high-risk communities [75-77]. At present, the use of reported signs and symptoms for screening of $S$. mansoni, however, has been only moderately successful and there remains a need for alternative approaches to be evaluated and validated.

Against this background, in 2001 the 54th World Health Assembly passed a resolution urging Member States to provide regular drug treatment to high-risk groups. In support of this important milestone in helminth control, WHO set a global target of regularly treating at least $75 \%$ of all school-age children. This goal has been given added impetus with the recent influx of major new sources of funding, notably from the Bill and Melinda Gates Foundation, which has, for example, supported the Schistosomiasis Control Initiative. The Schistosomiasis Control Initiative works with national governments and partners to ensure that treatment is available for at-risk groups in sub-Saharan Africa [78]. Jointly launched by the United Nations Educational, Scientific and Cultural Organization (UNESCO), the United Nations Children's Fund (UNICEF), WHO and the World Bank, the Focusing Resources on Effective School Health (FRESH) initiative also supports drug delivery through schools.

\section{Diagnosis}

The most commonly used method for parasitological diagnosis of schistome infec- tion is the detection of distinctive parasites in the faeces (S. mansoni) or urine (S. haematobium). Diagnosis can also be made on the detection of schistosome-specific antibodies $[79,80]$. The two most commonly employed immunological markers are circulating anodic antigens (CAA) and circulating cathodic antigens (CCA), using various assay methods. Recent results have suggested that ELISA-based detection of egg antigens offers a highly sensitive and specific method of diagnosis. Finally, the potential of PCR for detecting schistosome DNA in faecal matter is also being investigated [81]. For the assessment of morbidity, ultrasonography is a safe, rapid, noninvasive technique that enables clinical complications, such as hydronephrosis, bladder cancer and portal hypertension, to be easily assessed and the course after therapy to be monitored [68,71].

\section{Drugs}

Its broad spectrum of activity, excellent safety profile and high therapeutic efficacy means that praziquantel is the current drug of choice for the treatment of schistosomiasis $[82,83]$. Other drugs recommended in the treatment of schistosomiasis include metrifonate, which is active against $S$. haematobium and oxamniquine, which is effective against S. mansoni. Recently, however, the price of praziquantel has fallen considerably and because the price of oxamniquine has not decreased, praziquantel is replacing it. The decreasing demand for oxamniquine might stop oxamniquine production, as it already has for metrifonate.

The planned widespread use of praziquantel in human populations has raised concerns of the potential of drug resistance [84]. Recent lower than normal cure rates of S. mansoni infections in Senegal are probably due to very high re-infection rates

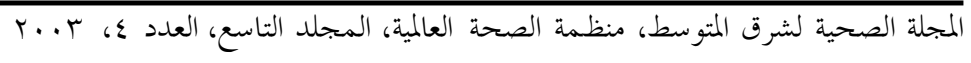


with a relatively high number of developing immature parasites not susceptible to praziquantel rather than resistance per se [85]. Certain schistosome isolates, nonetheless, may be less susceptible to praziquantel [86]. Despite the lack of conclusive evidence of drug resistance in humans, there remains a need for rigorous monitoring and surveillance of drug efficacy in ongoing control programmes, along with improved knowledge of the mechanisms of resistance $[84,86]$.

If praziquantel resistance develops, there will be new demands for anti-schistosomal drugs. Recently, artemisinin derivatives have been therapeutic against immature schistosomes and have been successful in clinical trials for S. japonicum in China [87]. Results from a randomized trial of artemether for chemotherapy against $S$. mansoni and $S$. haematobium show that a 6 $\mathrm{mg} / \mathrm{kg}$ dose had protective efficacies of $50 \%$ and $25 \%$, respectively $[87,88]$. A combination of praziquantel and artemether would be effective against all stages of the parasite in the human host [87]. However, artemether is expensive and widespread use of artemether in malarious regions would be unsuitable as it might contribute to the development of artemether resistant Plasmodium spp. Investigations are also under way to investigate the efficacy of praziquantel in combination with oxamniquine. Recent studies in Egypt have investigated the efficacy of Mirazid (Pharma, Alexandria, Egypt), an extract from the plant Commiphora molmol, or myrrh, in the treatment of schistosomiasis, but more clinical trials and laboratory studies are needed to adequately assess therapeutic efficacy [89].

\section{Vaccines}

Initial evidence that a human schistosomiasis vaccine might be possible was based on multiple lines of converging evidence including radiation-attenuated vaccines in experimental models and the occurrence of naturally acquired immunity in humans [90-92]. However, initial optimism stemming from the identification in the 1980s of the first vaccine candidate antigens that gave protection in animals faltered after disappointing results of independent trials of WHO designated schistosome vaccine candidates. These included the $S$. mansoni antigens paramyosin (Sm97), glutathioneS-transferase (Sm28), a fragment of myosin (rIrV5), triose-phosphate isomerase and the tegumental molecules Sm23 and Sm14. Unfortunately, none achieved sufficient levels of protection to proceed to phase III trials and the last decade has seen fewer new antigens identified [93]. Interestingly, experimental studies using attenuated vaccines have recently suggested that antigens relevant for the induction of immunity in this model are expressed by schistosomula during their migration from the skin to the lungs, and although such vaccines cannot be used in humans, they do provide a useful future paradigm for future research [94,95]. Other approaches under current investigation are the use of DNA vaccines [96]. Whereas the study of other parasites has benefited from the revolution in genomics and bioinformatics, the study of the schistosome genome has lagged behind. However, several new initiatives have been launched that hold promise for future vaccine development, including the use of genome sequencing, proteomics and micro-arrays [97].

\section{Control of transmission}

Although drug treatment and any future vaccines will help reduce disease due to schistosomiasis, they will not limit the reinfections that rapidly occur in endemic populations. Consequently, chemotherapy

المجلة الصحية لشرق المتوسط، منظمة الصحة العالمية، المجلد التاسع، العدد ع، ب... 
must be combined with transmission control efforts. Mollusciciding, commonly through the use of niclosamide, to kill intermediate host populations was previously used, but its prohibitive cost, potential environment-damaging effects and the occurrence of resistance have limited its widespread use today. Other methods including environmental modifications and biological control have limited applicability.

Undoubtedly sanitation and hygiene are essential for the long-term control of schistosomiasis. The length of time required for sanitation to improve and behaviour to change necessitates a need for a quick- acting, medium-term measure to control helminth infections, namely chemotherapy. Health education programmes and improvements in water and sanitation systems are necessary to ensure the sustainability of current drug programmes [98].

\section{Acknowledgements}

S. Brooker is supported by a Wellcome Trust Advanced Training Fellowship and L. Vivas by the Medicines for Malaria Venture.

\section{References}

1. The world health report 2003-shaping the future. Geneva, World Health Organization, 2003 (http://www.who.int/whr/ 2003/en/, accessed 23 June 2004).

2. Strategic direction for research. Geneva, Special Programme for Research and Training in Tropical Diseases, 2002 (http://www.who.int/tdr/diseases/malaria/direction.htm, accessed 23 June 2004.

3. Sachs JD. A new global effort to control malaria. Science, 2002, 298(5591): 122-4.

4. Greenwood B et al. Malaria in 2002. Nature, 2002, 415(6872):670-2.

5. Goodman CA et al. Cost-effectiveness of malaria control in sub-Saharan Africa. Lancet, 1999, 354(9176):378-85.

6. Murray CK et al. Rapid diagnostic testing for malaria. Tropical medicine and international health, 2003, 8(10):876-83.

7. Gascoyne P et al. Microfluidic approaches to malaria detection. Acta tropica, 2004, 89(3):357-69.

8. Piper $\mathrm{R}$ et al. Immunocapture diagnostic assays for malaria using plasmodium lactate dehydrogenase (pLDH). American journal of tropical medicine and hygiene, 1999, 60(1):109-18.

9. Moreno A et al. Use of the DELI-microtest to determine the drug sensitivity of Plasmodium falciparum in Burkina Faso. Annals of tropical medicine and parasitology, 2001, 95(3):309-12.

10. Moody A. Rapid diagnostic tests for malaria parasites. Clinical microbiology reviews, 2002, 15(1):66-78.

11. Palmer CJ et al. Multicenter study to evaluate the OptiMAL test for rapid diagnosis of malaria in U.S. hospitals. Journal of clinical microbiology, 2003, 41(11):5178-82.

12. Huong NM et al. Comparison of three antigen detection methods for diagnosis and therapeutic monitoring of malaria: a field study from southern Vietnam. Tropical medicine and international health, 2002, 7(4):304-8.

13. Forney JR et al. Devices for rapid diagnosis of Malaria: evaluation of prototype assays that detect Plasmodium falciparum histidine-rich protein 2 and a Plasmodium vivax-specific antigen. Journal

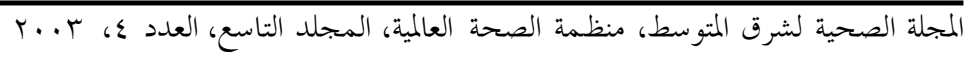


of clinical microbiology, 2003, 41(6): 2358-66.

14. Wongsrichanalai $C$ et al. Rapid diagnostic devices for malaria: field evaluation of a new prototype immunochromatographic assay for the detection of Plasmodium falciparum and non-falciparum Plasmodium. American journal of tropical medicine and hygiene, 2003, 69(1): 26-30.

15. Toure $Y T$ et al. The challenges of doing more against malaria, particularly in Africa. Bulletin of the World Health Organization, 2000, 78(12):1376.

16. Maxwell CA et al. Variation of malaria transmission and morbidity with altitude in Tanzania and with introduction of alphacypermethrin treated nets. Malaria journal, 2003, 2(1):28.

17. Howard $\mathrm{N}$ et al. Socio-economic factors associated with the purchasing of insecticide-treated nets in Afghanistan and their implications for social marketing. Tropical medicine and international health, 2003, 8(12):1043-50.

18. Guyatt $H L$ et al. Too poor to pay: charging for insecticide-treated bednets in highland Kenya. Tropical medicine and international health, 2002, 7(10):846-50.

19. Onwujekwe $O$ et al. Inequalities in purchase of mosquito nets and willingness to pay for insecticide-treated nets in Nigeria: challenges for malaria control interventions. Malaria journal, 2004, 3(1): 6 .

20. Africa malaria report 2003. Geneva, United Nations Children's Fund and the World Health Organization, 2003 (http:// www.rbm.who.int/amd2003/amr2003/ amr_toc.htm, accessed 23 June 2004).

21. Bhatia MR et al. Cost-effectiveness of malaria control interventions when malaria mortality is low: insecticide-treated nets versus in-house residual spraying in India. Social science and medicine, 2004, 59(3):525-39.

22. Guerin PJ et al. Malaria: current status of control, diagnosis, treatment, and a proposed agenda for research and development. Lancet infectious diseases, 2002, 2(9):564-73.

23. Hoffman SL et al. Protection of humans against malaria by immunization with radiation-attenuated Plasmodium falciparum sporozoites. Journal of infectious diseases, 2002, 185(8):1155-64.

24. Luke TC et al. Rationale and plans for developing a non-replicating, metabolically active, radiation-attenuated Plasmodium falciparum sporozoite vaccine. Journal of experimental biology, 2003, 206(Pt 21):3803-8.

25. Butler D. Mosquito production mooted as fast track to malaria vaccine. Nature, 2003, 425(6957):437.

26. Bojang KA et al. Efficacy of RTS,S/AS02 malaria vaccine against Plasmodium falciparum infection in semi-immune adult men in the Gambia: a randomised trial. Lancet, 2001, 358(9297):1927-34.

27. Moorthy VS, Good HF, Hill AV. Malaria vaccine developments. Lancet, 2004, 363(9403):150-6.

28. Epstein JE et al. Safety, tolerability and antibody responses in humans after sequential immunization with a PfCSP DNA vaccine followed by the recombinant protein vaccine RTS,S/AS02A. Vaccine, 2004, 22(13-14):1592-603.

29. Attaran A et al. WHO, the Global Fund, and medical malpractice in malaria treatment. Lancet, 2004, 363(9404):237-40.

30. McGready $\mathrm{R}$ et al. Artemisinin antimalarials in pregnancy: a prospective treatment study of 539 episodes of multidrug-resistant Plasmodium falci-

المجلة الصحية لشرق المتوسط، منظمة الصحة العالمية، المجلد التاسع، العدد ع، ب... 
parum. Clinical infectious diseases, 2001, 33(12):2009-16.

31. Nosten $\mathrm{F}$ et al. Burden of malaria during pregnancy in areas of stable and unstable transmission in Ethiopia during a nonepidemic year. Journal of infectious diseases, 2003, 188(8):1259-61; author reply $1561-2$.

32. Schellenberg $D$ et al. Intermittent treatment for malaria and anaemia control at time of routine vaccinations in Tanzanian infants: a randomised, placebo-controlled trial. Lancet, 2001, 357(9267): 1471-7.

33. Trouiller $P$ et al. Drug development for neglected diseases: a deficient market and a public-health policy failure. Lancet, 2002, 359(9324):2188-94.

34. MMV projects. Geneva, Medicines for Malaria Venture, 2003 (http://www.mmv. org/pages/content_frame.asp? ThePage=page1_0003_1 .htm \&Nav= 0003 , accessed $2 \overline{6}$ June 2004 ).

35. Nwaka S et al. Virtual drug discovery and development for neglected diseases through public-private partnerships. Nature reviews. Drug discovery, 2003, 2(11):919-28.

36. Leishmaniasis. Geneva, Special Programme for Research and Training in Tropical Diseases, 2002 (http://www. who.int/tdr/diseases/leish/diseaseinfo. htm, accessed 23 June 2004).

37. Desjeux P. The increase in risk factors for leishmaniasis worldwide. Transactions of the Royal Society of Tropical Medicine and Hygiene, 2001, 95(3):239-43.

38. Berhe $\mathrm{N}$ et al. HIV viral load and response to antileishmanial chemotherapy in co-infected patients. AIDS, 1999, 13(14):1921-5.

39. Cruz I et al. Leishmania in discarded syringes from intravenous drug users. Lancet, 2002, 359(9312):1124-5.
40. Herwaldt BL. Leishmaniasis. Lancet, 1999, 354(9185):1191-9.

41. Guerin PJ et al. Visceral leishmaniasis: current status of control, diagnosis, and treatment, and a proposed research and development agenda. Lancet infectious diseases, 2002, 2(8):494-501.

42. Boelaert $M$ et al. A comparative study of the effectiveness of diagnostic tests for visceral leishmaniasis. American journal of tropical medicine and hygiene, 2004, 70(1):72-7.

43. Sundar S. Drug resistance in Indian visceral leishmaniasis. Tropical medicine and international health, 2001, 6(11): 849-54.

44. Lira $\mathrm{R}$ et al. Evidence that the high incidence of treatment failures in Indian kala-azar is due to the emergence of antimony-resistant strains of Leishmania donovani. Journal of infectious diseases, 1999, 180(2):564-7.

45. Thakur CP et al. Amphotericin B deoxycholate treatment of visceral leishmaniasis with newer modes of administration and precautions: a study of 938 cases. Transactions of the Royal Society of Tropical Medicine and Hygiene, 1999, 93(3):319-23.

46. Sundar $S$ et al. Single-dose liposomal amphotericin B in the treatment of visceral leishmaniasis in India: a multicenter study. Clinical infectious diseases, 2003, 37(6):800-4.

47. Sundar S et al. Oral miltefosine for Indian visceral leishmaniasis. New England journal of medicine, 2002, 347(22): 1739-46.

48. Thakur CP et al. A prospective randomised, comparative, open-label trial of the safety and efficacy of paromomycin (aminosidine) plus sodium stibogluconate versus sodium stibogluconate alone for the treatment of visceral leish- 
maniasis. Transactions of the Royal Society of Tropical Medicine and Hygiene, 2000, 94(4):429-31.

49. De La Rosa R et al. Incidence of and risk factors for symptomatic visceral leishmaniasis among human immunodeficiency virus type 1-infected patients from Spain in the era of highly active antiretroviral therapy. Journal of clinical microbiology, 2002, 40(3):762-7.

50. Garnier T et al. Topical treatment for cutaneous leishmaniasis. Current opinion in investigational drugs, 2002, 3(4):53844.

51. Asilian A et al. Treatment of cutaneous leishmaniasis with aminosidine (paromomycin) ointment: double-blind, randomized trial in the Islamic Republic of Iran. Bulletin of the World Health Organization, 2003, 81(5):353-9.

52. Arevalo I et al. Successful treatment of drug-resistant cutaneous leishmaniasis in humans by use of imiquimod, an immunomodulator. Clinical infectious diseases, 2001, 33(11):1847-51.

53. Brodskyn $\mathrm{C}$ et al. Vaccines in leishmaniasis: advances in the last five years. Expert review of vaccines, 2003, 2(5): 705-17.

54. Gavgani AS et al. Effect of insecticideimpregnated dog collars on incidence of zoonotic visceral leishmaniasis in Iranian children: a matched-cluster randomised trial. Lancet, 2002, 360(9330): 374-9.

55. Stich A et al. Human African trypanosomiasis. British medical journal, 2002, 325(7357):203-6.

56. Barrett MP et al. The trypanosomiases. Lancet, 2003, 362(9394):1469-80.

57. Fevre EM et al. The origins of a new Trypanosoma brucei rhodesiense sleeping sickness outbreak in eastern Uganda. Lancet, 2001, 358(9282):625-8.
58. Simarro PP et al. Attitude towards CATTpositive individuals without parasitological confirmation in the African Trypanosomiasis (T.b. gambiense) focus of Quicama (Angola). Tropical medicine and international health, 1999, 4(12): 858-61.

59. Papadopoulos MC et al. A novel and accurate diagnostic test for human African trypanosomiasis. Lancet, 2004, 363 (9418):1358-63.

60. Barry JD et al. Antigenic variation in trypanosomes: enhanced phenotypic variation in a eukaryotic parasite. Advances in parasitology, 2001, 49:1-70.

61. Legros $D$ et al. Treatment of human African trypanosomiasis - present situation and needs for research and development. Lancet infectious diseases, 2002, 2(7):437-40.

62. Burri C et al. Efficacy of new, concise schedule for melarsoprol in treatment of sleeping sickness caused by Trypanosoma brucei gambiense: a randomised trial. Lancet, 2000, 355(9213):1419-25.

63. Pepin J et al. Short-course eflornithine in Gambian trypanosomiasis: a multicentre randomized controlled trial. Bulletin of the World Health Organization, 2000, 78(11):1284-95.

64. Grant IF. Insecticides for tsetse and trypanosomiasis control: is the environmental risk acceptable? Trends in parasitology, 2001, 17(1):10-4.

65. Awasthi $S$ et al. Helminthic infections. British medical journal, 2003, 327 (7412):431-3.

66. Jukes MC et al. Heavy schistosomiasis associated with poor short-term memory and slower reaction times in Tanzanian schoolchildren. Tropical medicine and international health, 2002, 7(2):104-17.

67. McGarvey ST et al. Child growth, nutritional status, and schistosomiasis

المجلة الصحية لشرق المتوسط، منظمة الصحة العالمية، المجلد التاسع، العدد ع، ب... 
japonica in Jiangxi, People's Republic of China. American journal of tropical medicine and hygiene, 1993, 48(4): 547-53.

68. Hatz CF. The use of ultrasound in schistosomiasis. Advances in parasitology, 2001, 48:225-84.

69. Boisier $\mathrm{P}$ et al. Reversibility of Schistosoma mansoni-associated morbidity after yearly mass praziquantel therapy: ultrasonographic assessment. Transactions of the Royal Society of Tropical Medicine and Hygiene, 1998, 92(4): 451-3.

70. Frenzel $\mathrm{K}$ et al. Evidence for a long-term effect of a single dose of praziquantel on Schistosoma mansoni-induced hepatosplenic lesions in northern Uganda. American journal of tropical medicine and hygiene, 1999, 60(6): 927-31.

71. Richter J. The impact of chemotherapy on morbidity due to schistosomiasis. Acta tropica, 2003, 86(2-3):161-83.

72. McGarvey ST et al. Schistosomiasis japonica and childhood nutritional status in northeastern Leyte, the Philippines: a randomized trial of praziquantel versus placebo. American journal of tropical medicine and hygiene, 1996, 54(5):498-502.

73. Nokes $\mathrm{C}$ et al. Evidence for an improvement in cognitive function following treatment of Schistosoma japonicum infection in Chinese primary schoolchildren. American journal of tropical medicine and hygiene, 1999, 60(4): 556-65.

74. Savioli $L$ et al. Schistosomiasis and soiltransmitted helminth infections: forging control efforts. Transactions of the Royal Society of Tropical Medicine and Hygiene, 2002, 96(6):577-9.

75. Hall $A$ et al. Alternatives to bodyweight for estimating the dose of praziquantel needed to treat schistosomiasis. Trans- actions of the Royal Society of Tropical Medicine and Hygiene, 1999, 93(6): 653-8.

76. Montresor A et al. Development and validation of a 'tablet' pole for the administration of praziquantel in sub-Saharan Africa. Transactions of the Royal Society of Tropical Medicine and Hygiene, 2001, 95(5):542-4.

77. Lengeler $\mathrm{C}$ et al. Screening for schistosomiasis with questionnaires. Trends in parasitology, 2002, 18(9):375-7.

78. Schistosomiasis control initiative. London, Department of Infectious Disease Epidemiology, Imperial College London, 2004 (http://www.schisto.org, accessed 28 June 2004).

79. Hamilton JV et al. Diagnosis of schistosomiasis: antibody detection, with notes on parasitological and antigen detection methods. Parasitology, 1998, 117 (suppl.): S41-57.

80. Doenhoff MJ et al. Specific and sensitive diagnosis of schistosome infection: can it be done with antibodies? Trends in parasitology, 2004, 20(1):35-9.

81. Pontes LA et al. Detection by polymerase chain reaction of Schistosoma mansoni DNA in human serum and feces. American journal of tropical medicine and hygiene, 2002, 66(2):157-62.

82. Fenwick $A$ et al. Drugs for the control of parasitic diseases: current status and development in schistosomiasis. Trends in parasitology, 2003, 19(11):509-15.

83. Utzinger $\mathrm{J}$ et al. Schistosomiasis and soil-transmitted helminthiasis: common drugs for treatment and control. Expert opinions in pharmacotherapy, 2004, 5(2):263-85.

84. Albonico M. Methods to sustain drug efficacy in helminth control programmes. Acta tropica, 2003, 86(2-3):233-42. 
85. Danso-Appiah A et al. Interpreting low praziquantel cure rates of Schistosoma mansoni infections in Senegal. Trends in parasitology, 2002, 18(3):125-9.

86. Hagan $P$ et al. Schistosomiasis control: keep taking the tablets. Trends in parasitology, 2004, 20(2):92-7.

87. Utzinger $\mathrm{J}$ et al. Current progress in the development and use of artemether for chemoprophylaxis of major human schistosome parasites. Current medicinal chemistry, 2001, 8(15):1841-60.

88. Utzinger $\mathrm{J}$ et al. Oral artemether for prevention of Schistosoma mansoni infection: randomised controlled trial. Lancet, 2000, 355(9212):1320-5.

89. El Baz MA et al. Clinical and parasitological studies on the efficacy of Mirazid in treatment of schistosomiasis haematobium in Tatoon, Etsa Center, El Fayoum Governorate. Journal of the Egyptian Society of Parasitology, 2003, 33(3):76176.

90. Coulson PS. The radiation-attenuated vaccine against schistosomes in animal models: paradigm for a human vaccine? Advances in parasitology, 1997, 39: 271-336.

91. Wynn TA et al. Defining a schistosomiasis vaccination strategy - is it really Th1 versus Th2? Parasitology today, 2000, 16(11):497-501.
92. Hagan $P$ et al. Schistosomiasis vaccines. Expert opinion on biological therapy, 2003, 3(8):1271-8.

93. Pearce EJ. Progress towards a vaccine for schistosomiasis. Acta tropica, 2003, 86(2-3):309-13.

94. Mountford AP et al. Interleukin-12 and protective immunity to schistosomes. Brazilian journal of medical and biological research, 1998, 31(1):163-9.

95. Wilson RA et al. Strategies for a schistosome vaccine: can we manipulate the immune response effectively? Microbes and infection, 1999, 1(7):535-43.

96. Da'Dara AA et al. A DNA-prime/proteinboost vaccination regimen enhances Th2 immune responses but not protection following Schistosoma mansoni infection. Parasite immunology, 2003, 25(8-9):429-37.

97. The Schistosoma mansoni Genome Project. Hinxton, Cambridge, United Kingdom, The Wellcome Trust Sanger Institute, 2003 (http://www.sanger.ac.uk/ Projects/S_mansoni/, accessed 28 June 2004).

98. Utzinger $\mathrm{J}$ et al. Sustainable schistosomiasis control-the way forward. Lancet, 2003, 362(9399):1932-4.

المجلة الصحية لشرق المتوسط، منظمة الصحة العالمية، المجلد التاسع، العدد ع، ب... 